\title{
Laparoscopic totally extraperitoneal inguinal hernia repair versus open Lichtenstein hernioplasty: A randomized trial
}

\author{
Ahmad Mohammed Ibrahim, MD \\ Department of General Surgery, Ain-Shams University, Cairo, Egypt
}

\begin{abstract}
Surgical techniques of inguinal hernia repair have been evolving over the past decade. The present study was conducted to compare the outcomes of laparoscopic totally extraperitoneal inguinal hernia repair (TEP) and open Lichtenstein hernioplasty. A total of 72 patients with 80 inguinal hernias were recruited between April 2004 and February 2007, and were randomized to undergo either TEP or open Lichtenstein hernioplasty under general anesthesia. Group I consisted of 32 patients who underwent TEPs (bilateral repairs in 8 patients) while Group II comprised 40 patients who underwent unilateral open Lichtenstein hernioplasties. A prospective collection and analysis of data were performed. Only 2 patients in group 1 were converted to open surgery. The mean operation time for TEP $(52 \pm 17.2 \mathrm{~min})$ was significantly shorter than for open Lichtenstein hernioplasty $(64 \pm 15.6 \mathrm{~min})(p<0.001)$. The pain score at rest was significantly lower in the TEP group than in the open group on postoperative days $0,1,4,5$, and 6. The mean length of hospital stay following laparoscopic and open repairs was 1.8 and 2.2 days respectively $(P>0.05)$. On the average, the patients returned to work 8.2 days after TEP and 16.4 days after Lichtenstein hernioplasty $(p<0.05)$. Postoperative recovery and morbidity rates were otherwise comparable between the two groups. The incidence of chronic groin pain 1 year after TEP (9.4\%) was significantly lower than after open surgery (22.6\%) ( $p$ $<0.05)$. None of the patients in either group showed recurrence at the last follow-up assessment.

In conclusion; postoperative recovery, morbidity rates and recurrence rates were comparable between the two groups. However TEP was superior to open Lichtenstein hernioplasty with regards to postoperative pain, time to return to work, and incidence of chronic groin pain.
\end{abstract}

\section{Introduction:}

Inguinal hernia repair is one of the commonest surgical operations in the world. ${ }^{1}$ Over the past decade, a tremendous variety of surgical techniques have been employed in the repair of inguinal hernias. Currently, open anterior tension-free mesh repair (Lichtenstein) is the most frequently performed hernia procedure, but laparoscopic preperitoneal repairs are gaining in popularity. ${ }^{2,3}$

The technique of laparoscopic inguinal hernia repair has evolved from the early transperitoneal route to a totally extraperitoneal approach (TEP), which avoids the development of port site hernia and bowel adhesions. ${ }^{4}$ TEP combines the principles of open posterior preperitoneal repair with the advantages of minimally invasive surgery. 5 Posterior preperitoneal approach avoids damaging the external oblique muscle and thus preserves the shutter mechanism of the inguinal canal. 6 In recent years, an increasing emphasis has been placed on the long-term outcomes for inguinal hernia repair, particularly the risk of chronic groin pain. 7,8

The current clinical trial was undertaken to compare the perioperative complications and 1-year outcomes of TEP and open Lichtenstein hernioplasty for the repair of inguinal hernia and to evaluate the justification for laparoscopic hernia repair.

\section{Patients and methods:}

A total of 72 patients with 80 inguinal hernias were recruited between April 2004 and February 2007. They were randomized to have either TEP or open Lichtenstein hernioplasty under general anesthesia. Group I consisted of 32 patients with 40 inguinal hernias who underwent TEPs (bilateral repairs in 8 patients) 
while Group II comprised 40 patients who underwent unilateral open Lichtenstein hernioplasties.

All the patients were admitted on the day of operation after fasting from midnight. Hernia types were determined intraoperatively according to the Nyhus classification. ${ }^{9}$ All perioperative and postoperative data were prospectively collected and entered into a computer database. All operations were performed by the author in standardized surgical steps.

\section{Operative technique - Laparoscopic extraperitoneal repair:}

The laparoscopic totally extraperitoneal inguinal hernia repair (TEP) procedure was performed like described by Tamme et al. ${ }^{3}$ Patients emptied their urinary bladder and received a diclofenac suppository $(50 \mathrm{mg}$ ) before surgery. Urinary catheterization and balloon dissection of the preperitoneal space were not used. A transverse subumbilical incision was made to expose the anterior rectus sheath on the side of the inguinal hernia. The anterior rectus sheath was divided transversely to expose the rectus muscle, which was retracted laterally. A 10-mm blunt-tip reusable trocar was inserted into the preperitoneal space and insufflated with carbon dioxide to a pressure of $10 \mathrm{mmHg}$. Under direct endoscopic vision, a 5-mm reusable trocar port was placed at midline, about $8 \mathrm{~cm}$ from the pubis. The extraperitoneal space was dissected and created by endoscissors with diathermy. Another 5$\mathrm{mm}$ reusable trocar was inserted at the anterior axillary line, about $3 \mathrm{~cm}$ proximal to the ipsilateral anterosuperior iliac spine.

For direct inguinal hernia, after reduction of the peritoneal hernial sac Figure(1), the attenuated transversalis fascia was routinely inverted and ligated with an endoloop if feasible. The indirect peritoneal sac was isolated and ligated with absorbable suture followed by distal transection using endoscissors. The spermatic cord and pelvic floor were parietalized for a length of at least $4 \mathrm{~cm}$ Figure(2). A 10x14 $\mathrm{cm}^{2}$ Prolene mesh (Prolene mesh; Ethicon Ltd, Somerville, NJ, USA) was introduced to cover the posterior wall of the inguinal canal Figure(3), obturator foramen, and femoral and internal inguinal rings. The mesh was anchored in place with an endostapler Figure(4) only if the maximal diameter of the hernial defect exceeded $4 \mathrm{~cm}$. The three trocar wounds were infiltrated with $10 \mathrm{ml}$ of $0.5 \%$ bupivacaine altogether before closure.

\section{Operative technique - Open Lichtenstein hernioplasty:}

The Lichtenstein hernioplasty technique followed the principles described by Lichtenstein et al. ${ }^{10}$ An oblique groin incision of about $6-8 \mathrm{~cm}$ long was made. After identification of superficial inguinal ring, the inguinal canal was opened and the hernial sac was isolated. For indirect inguinal hernia, the peritoneal sac was routinely ligated and extirpated. For direct inguinal hernia, the hernial sac was inverted and closed with purse-string suture over the transversalis fascia. The posterior wall of the inguinal canal was then reinforced by the placement of a laterally split Prolene mesh of about $8 \times 6 \mathrm{~cm}^{2}$. The mesh was fixed to the inguinal ligament and conjoint tendon with interrupted $2 / 0$ Prolene sutures.

\section{Post-operative management:}

All the patients were prescribed the oral paracetamol $500 \mathrm{mg}$ four times daily and diclofenac sodium SR 100mg daily upon request. They were allowed to resume diet and normal activities after the operation. The patients were discharged once they had micturated and ambulated comfortably. All the patients were followed up at the hernia clinic 1 week after discharge and at 12 months thereafter if the patient had an uneventful recovery. All complications were recorded during follow-ups.

The primary outcomes measures included severity of postoperative pain, time until return to work, incidence of chronic pain, and hernia recurrence at 1 year.

The severity of pain at rest and during coughing was assessed by linear analog pain score on a scale of 0 to 10 before discharge. Patients were taught to fill in a daily pain score chart and document the number of analgesic tablets taken at home. Chronic pain was assessed by interview at the clinic using a standard questionnaire during a follow-up 
assessment 1 year after the operation ${ }^{11}$. Time until return to work was calculated for the patients who were actively employed, whereas unemployed and retired patients were excluded. Total amount of analgesic consumption was based on the total number of analgesic tablets consumed by the patient during the hospital stay and at home. Recurrence of hernia was defined as reducible inguinal swelling, as demonstrated clinically.

Secondary outcome parameters included operation time, hospital stay, unanticipated admission, and morbidity rates. Operation time referred to the time from skin incision to the last suture. Length of hospital stay referred to

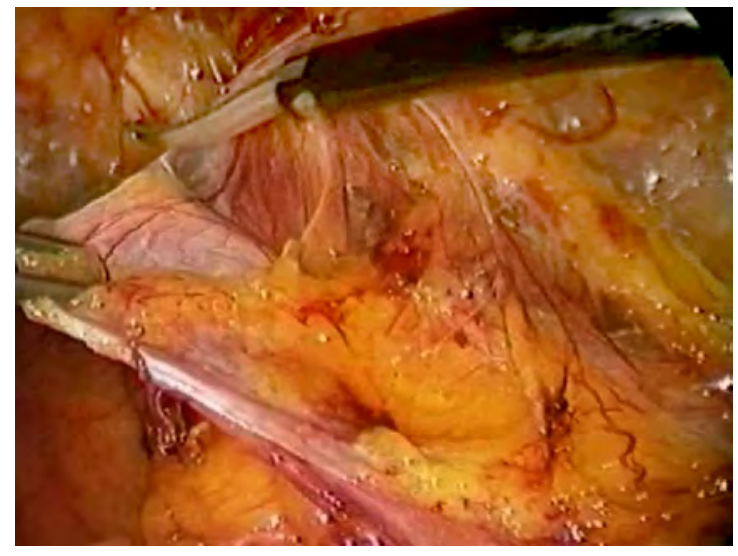

Figure (1): Reduction of the peritoneal hernial sac.

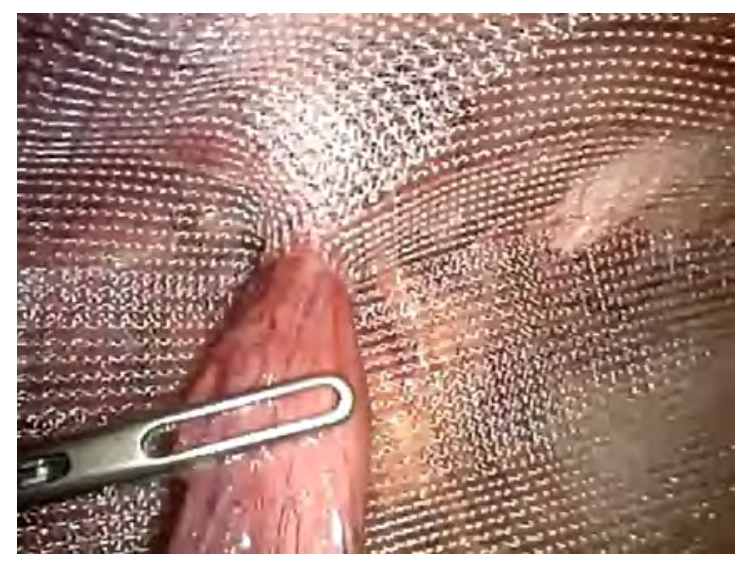

Figure (3): A 10x14 $\mathrm{cm}^{2}$ Prolene mesh was introduced to cover the posterior wall of the inguinal canal. the total number of nights spent in hospital after operation.

\section{Statistical analysis:}

Statistical analysis was performed with the help of computer software (SPSS/PC+ 7.0, SPSS, Chicago, Illinois, USA). Student's t test and chi-square test were used to detect differences between the two groups of patients. A "P" value of less than 0.05 was considered statistically significant. Values were expressed as mean \pm standard error of the mean (S.E.M.).

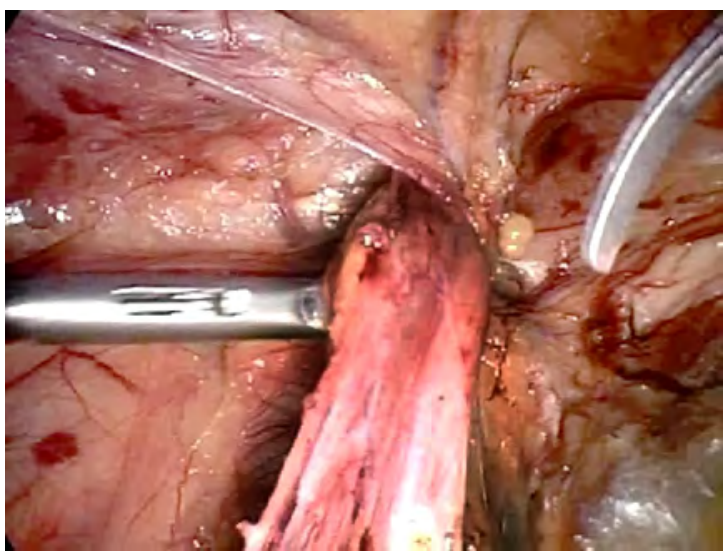

Figure (2): The spermatic cord was parietalized for a distance of at least 3 to $4 \mathrm{~cm}$.

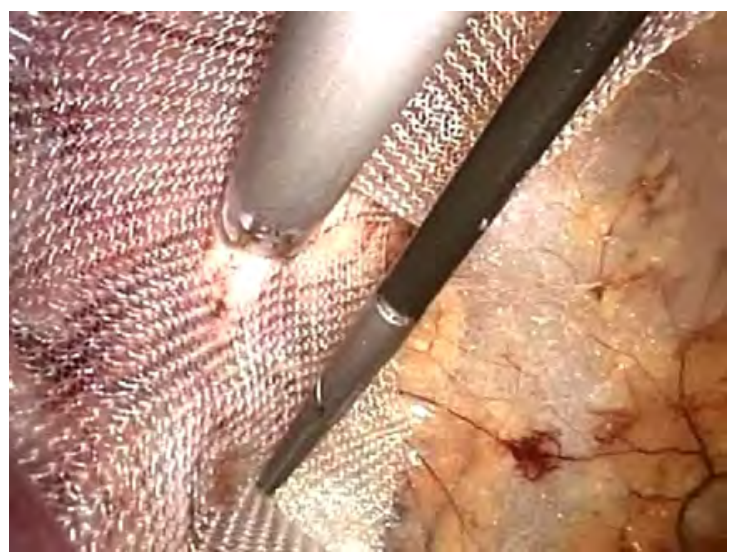

Figure (4): The mesh was anchored in place with an endostapler. 


\section{Results:}

The mean age of the study population $(\mathrm{n}=72)$ was $69 \pm 12$ (S.D.) years. Nyhus classification of the hernias were types II $(n=32)$, IIIA $(n=37)$, IIIB $(n=6)$, IVA $(n=2)$, IVB $(n=2)$ and IVD $(n=1)$. The demographic features of the two groups of patients are presented in Table(1).

Of the 32 laparoscopic extraperitoneal hernioplasties, two were converted to open operation because of adhesions and the presence of bowel within the peritoneal sac. The hernia anatomy of the two converted cases were sliding inguinal hernia $(\mathrm{n}=1)$ and recurrent inguinal hernia $(n=1)$.

The mean operation time for unilateral TEP, $52 \pm 17.2 \mathrm{~min}$, was significantly shorter than for open Lichtenstein hernioplasty $(64 \pm 15.6$ min; $p<0.001)$. For both groups of patients, there was no intra-operative complication or hospital mortality. Table(2) compares the postoperative complications between the two groups of patients. Comparison of recovery variables, including time to ambulation, micturition, and discharge, showed no significant difference between the two groups Table(3).

The pain score at rest for the TEP group was significantly lower than for the open group on postoperative days $0,1,4,5$ and 6 (p < 0.05 ), but comparison of the daily pain scores for coughing showed no significant difference between the two groups during the entire postoperative period.
The intraoperative and postoperative analgesic requirements were comparable between the two groups.

The mean length of hospital stay following laparoscopic and open repairs were $1.8 \pm 0.28$ days and $2.2 \pm 0.34$ days respectively $(\mathrm{P}<0.05)$.

A total of 58 patients had completed the interview and chronic pain questionnaire 1 year after the operation. They included 27 patients in the TEP group (22 patients with unilateral hernia and 5 patients with bilateral hernia) and 31 patients in the open group.

The incidence of chronic pain after open repair at 1 year $(22.6 \%, \mathrm{n}=7)$ was significantly higher than after TEP $(9.4 \%, \mathrm{n}=3 ; \mathrm{p}<0.05)$. The nature of chronic groin pain was mild, dull, and transient for all but one patient in the open group. None of the patients in either group had interference of their daily activities by the pain. A total of 6 patients ( 1 in the TEP group and 5 in the open group) reported a history of oral analgesic use for the chronic pain. Two patients in the open group experienced pain while lifting a heavy object or standing up from a squatting position, which exerted an adverse influence on their job performance. None of the patients were found to have clinical recurrence at the last followup assessment.

Table (1): Comparison of demographic features of the two groups of patients.

\begin{tabular}{|l|c|c|c|}
\hline & Group I & Group II & p Value \\
\hline Age (mean+S.D.) & $46 \pm 13.9$ & $51 \pm 9.6$ & $<0.05(\mathrm{NS})^{*}$ \\
\hline Sex (male:female) & $15: 1$ & $37: 3$ & $<0.05(\mathrm{NS}) \#$ \\
\hline Hernia anatomy\$ & & & $<0.05(\mathrm{NS}) \#$ \\
\hline II & 16 & 16 & \\
\hline IIIA & 17 & 20 & \\
\hline IIIB & 3 & 3 & \\
\hline IVA & 1 & 1 & \\
\hline IVB & 2 & 0 & \\
\hline IVD & 1 & 0 & \\
\hline
\end{tabular}

*Student's t-test \$Nyhus classification \#Chi-square test $\quad$ (NS) non significant 
Table (2): Post-operative complications of the 2 groups.

\begin{tabular}{|l|c|c|c|}
\hline Complications & Group I & Group II & p Value \\
\hline Seroma & 1 & 1 & $>0.05$ \\
\hline Hematoma & 1 & 0 & $>0.05$ \\
\hline Transient lateral thigh numbness & 1 & 0 & $>0.05$ \\
\hline Wound infection & 0 & 2 & $<0.05$ \\
\hline Retention of urine & 1 & 3 & $<0.05$ \\
\hline
\end{tabular}

Table (3): Comparison of recovery variables on the day of operation between the totally extraperitoneal inguinal hernioplasty (TEP) and open groups.

\begin{tabular}{|l|c|c|c|}
\hline Complications & Group I & Group II & p Value \\
\hline Time to ambulation (h) & $14.8 \pm 0.68$ & $18.4 \pm 0.52$ & $<0.05$ \\
\hline Time to micturition (h) & $3.7 \pm 0.85$ & $4.7 \pm 0.52$ & $<0.05$ \\
\hline Time to discharge (d) & $1.8 \pm 0.28$ & $2.2 \pm 0.34$ & $<0.05$ \\
\hline
\end{tabular}

\section{Discussion:}

The acceptance of laparoscopic hernia repair by general surgeons has been poor in early 1990s. ${ }^{12}$ It was mainly due to the evolving surgical technique and high recurrence rate in the early days. Other reasons included the cost of disposable instruments, potential serious complications and unavailability of long-term results. The technique of laparoscopic totally extraperitoneal repair (TEP) has now been refined and well-established. A number of randomized controlled trials have demonstrated the durable long-term outcome of TEP. ${ }^{13-16}$

The current study demonstrated that postoperative pain at rest after TEP was significantly less than after Lichtenstein hernioplasty. Similar findings also were demonstrated in most randomized trials comparing the outcomes of laparoscopic and open repair .3, 14, 16 Less post-operative pain allows patients to ambulate early with minimal discomfort and also results in a shorter period of convalescence. Although TEP involves a wide dissection of the preperitoneal space, the ilioinguinal and iliofemoral nerves, which are invariably encountered during anterior open repair, lie external to the preperitoneal plane. Injury to these sensory nerves during an anterior open repair probably accounts for the increased severity of postoperative pain and the increased incidence of chronic pain at 1 year. ${ }^{14}, 17$

Our findings demonstrated that the incidence of mild chronic pain after TEP was significantly lower than after open repair. Severe pain after inguinal hernia repair was rarely seen in our center. Kumar et al. ${ }^{8}$ reported that chronic pain was sufficiently severe to restrict daily physical or sporting activities in $18.1 \%$ of their patients.

Patients who underwent laparoscopic repairs regained their full physical performance much faster than those after conventional hernia repairs. ${ }^{3}$ Liem et al. also demonstrated a better quality of life in patients following TEPs in the recovery period, compared to those following conventional repairs. ${ }^{18}$

In the current study, the operation time for TEP was significantly shorter than for open repair. In a recent clinical trial, Colak et al. ${ }^{17}$ also reported similar findings. To achieve an average operation time of less than 1 hour for TEP of unilateral inguinal hernia, involved surgeons must have surmounted the steep learning curve for TEP. ${ }^{19}$ For bilateral and recurrent inguinal hernias, Mahon et al. ${ }^{20}$ also demonstrated a significantly shorter operation time for laparoscopic than for open repair.

With the escalating cost of medical care, more and more operations are being performed on an ambulatory basis. The shorter length of hospital stay in patients after laparoscopic 
hernia repairs confers economic advantages to the hospital, the patient and the society. The hospital saving from a reduced hospital stay could offset the cost of laparoscopic hernia repair. In a study by Heikkinen et al. ${ }^{21}$ open mesh repair was found to be cheaper for the hospital but the total costs for working patients were lower with the laparoscopic technique. Laparoscopic hernia repair was more effective and less expensive to the society. ${ }^{22}$ Ferzli et al. ${ }^{23}$ reported successful performance of laparoscopic hernia repair under local anesthesia on 10 patients but this technique remained experimental. Most recent reports of laparoscopic totally extraperitoneal repair had a low morbidity and recurrence rates. ${ }^{24-25}$ Wound infection was noticeably less frequent in patients after laparoscopic hernia repairs than those following open repairs. ${ }^{18}$

Recurrence was not detected in any of the patients in the current study. The recurrence rate for TEP has been proved superior to that for conventional suture herniorrhaphy. ${ }^{14,}$ 16, 17, 25 The chief reason for early recurrence after inguinal hernia repair, with either TEP or Lichtenstein hernioplasty, is technical or surgeon factors. Publications on long-term recurrence rates for TEP remain limited.

The patient's preference needs to be considered in any patient-centered quality service. More than $80 \%$ of our patients who had experience of both open and laparoscopic repairs would prefer to undergo laparoscopic operation again in the event of future recurrence. Our findings were consistent with a report by Kiruparan et al.; $93 \%$ of their patients with previous open hernia repair preferred laparoscopic hernia repair. ${ }^{26}$ These patients reported less pain and a rapid return to normal activity after laparoscopic operation.

\section{Conclusions:}

Both TEP and Lichtenstein hernioplasty are safe and efficacious techniques for the repair of inguinal hernia. The outcomes of laparoscopic totally extraperitoneal repair were superior to open Lichtenstein hernioplasty, with regard to the postoperative pain, time to return to work, and incidence of chronic groin pain. However the escalating cost of endoscopic procedures is a major disadvantage in our society.

The operative technique used should be a joint decision by the patient and the surgeon after the pros and cons of both approaches have been balanced.

\section{References:}

1- Rutkow IM: Epidemiologic, economic, and sociologic aspects of hernia surgery in the United States in the 1990s. Surg Clin North Am 1998; 78: 941-951.

2- Wright DM, Kennedy A, Baxter JN, Fullarton GM, Fife LM, Sunderland GT, O’Dwyer PJ: Early outcome after open versus extraperitoneal endoscopic tensionfree hernioplasty: A randomized clinical trial. Surgery 1996; 119: 552-557.

3- Tamme C, Scheidbach H, Hampe C, Schneider C, Kockerling F: Totally extraperitoneal endoscopic inguinal hernia repair (TEP): Results of 5,203 hernia repairs. Surg Endosc 2003; 17: 190-195.

4- Lucas SW, Arregui ME: Minimally invasive surgery for inguinal hernia. World J Surg 1999; 23: 350-355.

5- Ferzli G, Sayad P, Huie F, Hallak A, Usal $\mathrm{H}$ : Endoscopic extraperitoneal herniorrhaphy. A 5-year experience. Surg Endosc 1998; 12: 1311-1313.

6- Patino JF, Garcia Herreros LG, Zundel N: Inguinal hernia repair. The Nyhus posterior preperitoneal operation. Surg Clin North Am 1998; 78: 1063-1074.

7- Grant AM, Scott NW, O'Dwyer PJ: MRC Laparoscopic Groin Hernia Trial Group five-year follow-up of a randomized trial to assess pain and numbness after laparoscopic or open repair of groin hernia. Br J Surg 2004; 91: 1570-1574.

8- Kumar S, Wilson RG, Nixon SJ, Macintyre IM: Chronic pain after laparoscopic and open mesh repair of groin hernia. Br J Surg 2002; 89: 1476-1479.

9- Nyhus LM: Individualization of hernia repair: A new era. Surgery 1993; 114: 1-2.

10-Lichtenstein IL, Shulman AG, Amid PK, Montllor MM: The tension-free hernioplasty. Am J Surg 1989; 157: 188-193.

11-Lau H, Patil NG, Yuen WK, Lee F: Prevalence and severity of chronic groin 
pain following endoscopic totally extraperitoneal inguinal hernioplasty. Surg Endosc 2003; 17: 1620-1623.

12-Ooi LL, Koh J, Mack PO: Laparoscopic hernia repair in Singapore: Views from a cross-section of surgeons. Ann Acad Med Singapore 1996; 25: 643-645.

13-Juul P, Christensen K: Randomized clinical trial of laparoscopic versus open inguinal hernia repair. Br J Surg 1999; 86: 316-319.

14-Bringman S, Ramel S, Heikkinen TJ, Englund T, Westman B, Anderberg B: Tension-free inguinal hernia repair: TEP versus mesh-plug versus Lichtenstein: A prospective randomized controlled trial. Ann Surg 2003; 237: 142-147.

15-The MRC laparoscopic groin hernia trial group: Laparoscopic versus open repair of groin hernia: A randomized comparison. Lancet 1999; 354: 185-190.

16-Andersson B, Hallen M, Leveau P, Bergenfelz A, Westerdahl J: Laparoscopic extraperitoneal inguinal hernia repair versus open mesh repair: A prospective randomized controlled trial. Surgery 2003; 133: 464-472.

17-Colak T, Akca T, Kanik A, Aydin S: Randomized clinical trial comparing laparoscopic totally extraperitoneal approach with open mesh repair in inguinal hernia repair. Surg Laparosc Endosc Percutan Tech 2003; 13: 191-195.

18-Liem MS, van der Graaf Y, van Steensel CJ, Boelhouwer RU, Clevers GJ, Meijer WS, et al: Comparison of conventional anterior surgery and laparoscopic surgery for inguinal-hernia repair. $N$ Engl J Med 1997; 336: 1541- 1547.

19-Lau H, Patil NG, Yuen WK, Lee F: Learning curve for unilateral endoscopic totally extraperitoneal inguinal hernioplasty. Surg Endosc 2002; 16: 1724-1728.
20-Mahon D, Decadt B, Rhodes M: Prospective randomized trial of laparoscopic (transabdominal preperitoneal) vs open (mesh) repair for bilateral and recurrent inguinal hernia. Surg Endosc 2003; 17: 1386-1390.

21-Heikkinen TJ, Haukipuro K, Koivukangas P, Hulkko A: A prospective randomized outcome and cost comparison of totally extraperitoneal endoscopic hernioplasty versus Lichtenstein hernia operation among employed patients. Surg Laparosc Endosc 1998; 8: 338-344.

22-Kald A, Anderberg B, Carlsson P, Park PO, Smedh K: Surgical outcome and costminimisation-analyses of laparoscopic and open hernia repair: A randomized prospective trial with one year follow up. Eur J Surg 1997; 163: 505-510.

23-Ferzli G, Sayad P, Vasisht B: The feasibility of laparoscopic extraperitoneal hernia repair under local anesthesia. Surg Endosc 1999; 13: 588-590.

24-Bochkarev V, Ringley C, Vitamvas M, Oleynikov D: Bilateral laparoscopic inguinal hernia repair in patients with occult contralateral inguinal defects. Surg Endosc 2007; 21: 734-736.

25-Vidovic D, Kirac I, Glavan E, Ugura J, Ledinesky M, Beslin M: Laparoscopic totally extraperitoneal hernia repair versus open Lichtenstein hernia repair: Results and complications. J Lap Endo Adv Surg Tech 2007; 17(5): 585-590.

26-Kiruparan P, Pettit SH: Prospective audit of 200 patients undergoing laparoscopic inguinal hernia repair with followup from 1 to 4 years. J R Coll Surg Edinb 1998; 43: 13-16. 